\title{
Pregnancy's Relationship With Diabetes and Induced Hypertension: A Mini-Review
}

\author{
Ishita Saha ${ }^{1}$, Mahashweta Das ${ }^{2 *}$ \\ ${ }^{1}$ Department of Physiology, Calcutta Medical College and Hospital, Kolkata, West Bengal, India \\ ${ }^{2}$ Department of History, The University of Burdwan, Burdwan, West Bengal, India
}

Correspondence to: Mahashweta Das, Department of History, The University of Burdwan, Burdwan, West Bengal, India

Received date: November 24, 2021; Accepted date: December 5, 2021; Published date: December 12, 2021

Citation: Saha I, Das M (2021) Pregnancy's Relationship With Diabetes and Induced Hypertension: A Mini-Review. J Obst Gynecol Surg 2(2): pp. 1-4. doi: 10.52916/jogs214018

Copyright: (C2021 Saha I, et al. This is an open-access article distributed under the terms of the Creative Commons Attribution License, which permits unrestricted use, distribution and reproduction in any medium, provided the original author and source are credited.

\section{ABSTRACT}

It is pointed out in many articles that induced hypertension and gestational diabetes are well linked to pregnancy, but this relationship is not well determined. The report focuses on the pregnancy's relationship with diabetes and induced hypertension. It is found herein that mean pregnancy is directly related with glucose levels (GLUC) $(P=0.07)$, age $(P<0.01), B M I(P=0.07)$, Diabetic Women $(D W)$ status $(P=0.01)$, interaction effect of Triceps SkinFold Thickness (TSFT) and age (TSFT $\times$ Age) $(P<0.01)$, while it is inversely related with GLUC $\times$ Age $(P=0.02)$, TSFT $\times \mathrm{BMI}(\mathrm{P}<0.01)$, Insulin (INSU) $(\mathrm{P}=0.06)$ and Diabetes Pedigree Function (DPDF) $(\mathrm{P}=0.03)$. Variance of pregnancy is directly related with GLUC ( $P=0.07)$, Diastolic Blood Pressure (DBP) $(P=0.04)$, GLUC $\times$ TSFT $(P<0.01), D B P \times$ TSFT $(P<0.01)$, DPDF $(P=0.01)$, INSU $\times B M I(P=0.15)$, while it is inversely related with $G L U C \times D B P(P=0.01), T S F T(P<0.01)$, TSFT $\times$ DPDF $(P=0.03)$, INSU $(P=0.07)$. It is easily interpreted that pregnancy's mean is well connected to diabetic parameters such as INSU and GLUC levels, history of DW, DPDF, BMI, GLUC $\times$ Age, while its variance is connected to diabetic and hypertension parameters such as GLUC, DPDF, DBP, GLUC $\times$ TSFT, DBP $\times$ TSFT, INSU $\times$ BMI, GLUC $\times$ DBP, TSFT $\times$ DPDF, INSU. Moreover, it is derived that a diabetic female has a higher chance to be pregnant than a normal woman.

\section{Keywords:}

Diastolic Blood Pressure (DBP), Diabetes Pedigree Function (DPDF), Gestational diabete, Hypertension parameters, Joint Generalized Linear Gamma Models (JGLMS), Pregnancy

\section{Introduction}

There are some common problems such as hypertension and Gestational Diabetes (GD) disorders of pregnant women all over the whole world. Many articles have pointed out that GD disorders and hypertension are frequently noticed for pregnant women, while the relationships of pregnancy with GD disorders and hypertension are not recognized [1-5]. GD disorders can be stated if there is any dysglycemia that appears for the pregnancy's first time that is a global public health problem [3, 5, 6-9]. GD disorders is a primary cause for mothers' mortality and morbidity along with her neonate over the whole world [710]. In addition mothers are affected with induced hypertension during pregnancy $[6,9]$.

It is noted that many articles have pointed out that GD disorders and hypertension are linked to pregnancy. Most of the earlier articles tried to establish these linkages using simple correlation and regression, multiple regression, meta-analysis, etc. $[2,6,7,10]$. Here it is noted that pregnancy number is a discrete heterogeneous variable, which may not be modeled appropriately using simple and multiple regression analysis. Therefore, previous outcomes invite doubts and contradictions.

\section{Statistical Analysis}

It is noted herein that the pregnancy's number is the aimed response variable herein, which is a discrete form. In the total gestational period for a woman, the total number of fractional pregnancies within two months, called miss-carriage, can be considered a numerical value 0.5 . The considered misscarriages are known as natural abortions, while one can find induced abortions also [11-13]. This assumption turns the discrete variable pregnancy's number to a continuous variable. So, each pregnancy number is raised by 0.5 , which is simply the origin change of the data set. It is marked herein that the aimed response pregnancy's number is a heterogeneous variable, which is modeled herein using Joint Generalized Linear Models (JGLMs) that is well illustrated in the book by Lee et al. [14]. JGLMs are described in many research articles [15-17], which are not restated herein.

The current report searches the pregnancy's linkages to GD factors such as insulin levels ( $\mathrm{mu} \mathrm{U/ml}$ ) and glucose levels ( $\mathrm{mu}$ $\mathrm{U} / \mathrm{ml}$ ), DPDF, study unit's diabetic status (1=normal, $2=$ diabetic), $\mathrm{BMI}$, and hypertension risk factor DBP $(\mathrm{mm} \mathrm{Hg})$ along with TSFT(mm) and age (years), while the minimum subject's age is 21 years old, based on 768 gestational women. This data set is available on the website of $\mathrm{UCI}$ Machine Learning Repository. A heterogeneous response variable can be modeled using JGLMs under the log-normal or gamma distribution. Here the aimed response variable pregnancy's number is modeled using the log-normal JGLMs, and the summarized results are shown in Table 1.

The joint log-normal fitted models in Table 1 are verified by Figure 1, where Figure 1(a) reveals the absolute pregnancy's number fitted residuals plot with respect to the log-normal fitted predicted values, which is nearly a flat straight line, indicating that variance is constant with the running means. The other Figure 1(b) shows the mean pregnancy's number fitted 
Citation: Saha I, Das M (2021) Pregnancy's Relationship With Diabetes and Induced Hypertension: A Mini-Review. J Obst Gynecol Surg 2(2): pp. 1-4. doi: $10.52916 / j o g s 214018$

Table 1: Joint log-normal Pregnancy's number fitting mean and dispersion models.

\begin{tabular}{|c|c|c|c|c|c|}
\hline Model & Covariate & Estimate & Standard error & t-value & P-value \\
\hline \multirow[t]{11}{*}{ Mean } & Constant & -0.72 & 0.41 & -1.75 & 0.08 \\
\hline & GLUC & 0.01 & $<0.01$ & 1.91 & 0.07 \\
\hline & Age & 0.05 & 0.01 & 4.65 & $<0.01$ \\
\hline & GLUC $\times$ Age & -0.01 & $<0.01$ & -2.40 & 0.02 \\
\hline & TSFT & 0.01 & 0.01 & 0.09 & 0.93 \\
\hline & BMI & 0.01 & 0.01 & 1.81 & 0.07 \\
\hline & $\mathrm{TSFT} \times \mathrm{BMI}$ & -0.01 & 0.01 & -3.56 & $<0.01$ \\
\hline & INSU & -0.01 & 0.01 & -1.90 & 0.06 \\
\hline & DPDF & -0.19 & 0.09 & -2.15 & 0.03 \\
\hline & TSFT $\times$ Age & 0.01 & $<0.01$ & 4.69 & $<0.01$ \\
\hline & DW & 0.17 & 0.07 & 2.46 & 0.01 \\
\hline \multirow[t]{12}{*}{ Dispersion } & Constant & -1.73 & 0.85 & -2.04 & 0.04 \\
\hline & GLUC & 0.01 & 0.01 & 1.82 & 0.07 \\
\hline & DBP & 0.02 & 0.01 & 2.08 & 0.04 \\
\hline & GLUC × DBP & -0.01 & $<0.01$ & -2.79 & 0.01 \\
\hline & TSFT & -0.11 & 0.02 & -5.82 & $<0.01$ \\
\hline & GLUC $\times$ TSFT & 0.01 & $<0.01$ & 4.48 & $<0.01$ \\
\hline & $\mathrm{DBP} \times \mathrm{TSFT}$ & 0.01 & $<0.01$ & 4.05 & $<0.01$ \\
\hline & DPDF & 0.75 & 0.29 & 2.62 & 0.01 \\
\hline & $\mathrm{TSFT} \times \mathrm{DPDF}$ & -0.02 & 0.01 & -2.19 & 0.03 \\
\hline & INSU & -0.01 & 0.01 & -1.83 & 0.07 \\
\hline & BMI & 0.01 & 0.01 & 0.54 & 0.59 \\
\hline & $\mathrm{INSU} \times \mathrm{BMI}$ & 0.01 & $<0.01$ & 1.43 & 0.15 \\
\hline Dispersion & & \multicolumn{4}{|c|}{3478} \\
\hline
\end{tabular}

model of normal probability plot (Table 1), which does not are appropriate, which is supported by Figure 1. present any fitted discrepancy. So, the joint log-normal models

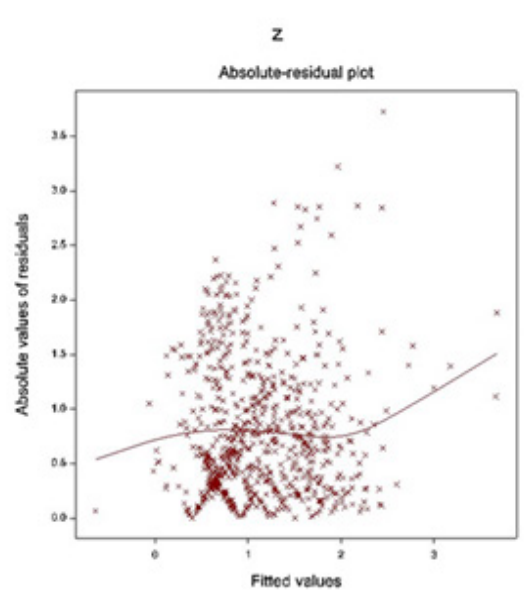

1(a)

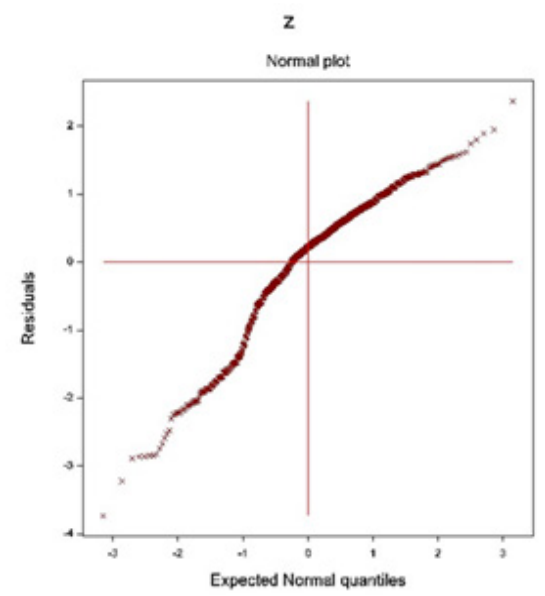

1(b)

Figure 1: For the joint log-normal Pregnancy's number fit (Table 1); (a) absolute residuals plot with respect to the fitted values; (b) the normal probability plot for the Pregnancy's number mean model. 


\section{Results}

All the derived summarized results from the joint log-normal pregnancy's number fitted models are displayed in Table 1. It is derived herein that mean pregnancy is directly related with GLUC levels $(P=0.07)$, age $(P<0.01)$, BMI $(P=0.07)$, DW status $(P=0.01)$, interaction effect TSFT $\times$ Age $(P<0.01)$, while it is inversely related with GLUC $\times$ Age $(P=0.02)$, TSFT $\times B M I(P<0.01)$, INSU $(P=0.06)$ and DPDF $(P=0.03)$. Variance of pregnancy is directly related with GLUC $(P=0.07), D B P(P=0.04), G L U C \times$ TSFT $(P<0.01)$, DBP $\times$ TSFT $(P<0.01)$, DPDF $(P=0.01)$, INSU $\times$ BMI $(P=0.15)$, while it is inversely related with GLUC $\times D B P(P=0.01)$, TSFT $(P<0.01)$, TSFT $\times$ DPDF $(P=0.03)$, INSU $(P=0.07)$.

JGL log-normal pregnancy's number fitted mean $\left(\mu^{\wedge}\right)$ model (Table 1 ) is

$\hat{\mu}=\exp (-0.72+0.01 \mathrm{GLUC}+0.05$ Age-0.01GLUC $\times$ Age+0.01TSFT+0. 01BMI-0.01TSFT $\times$ BMI-0.01INSU-0.19DPDF+0.01TSFT $\times$ Age +0.1 7DW),

and the JGL log-normal pregnancy's number fitted dispersion $\left(\hat{\sigma}^{2}\right)$ model (from Table 1$)$ is

$\hat{\sigma}^{2}=\exp (-1.73+0.01 \mathrm{GLUC}+0.02 \mathrm{DBP}-0.01 \mathrm{GLUC} \times \mathrm{DBP}-0.11 \mathrm{TSFT}+$ $0.01 \mathrm{GLUC} \times \mathrm{TSFT}+0.01 \mathrm{DBP} \times \mathrm{TSFT}+0.75 \mathrm{DPDF}-0.02 \mathrm{TSFT} \times$ DPDF0.01INSU+0.01BMI+0.01 INSU $\times$ BMI).

\section{Discussions}

The associations of pregnancy's number with diabetic functions can be noted from the derived mean model (Table 1). It is derived that mean pregnancy number is directly related with GLUC levels $(P=0.07)$, implying that it rises with the increase of GLUC levels. Mean pregnancy number is directly related with DW status ( $1=$ normal, $2=$ diabetic) $(P=0.01)$, revealing that pregnancy is higher for diabetic women than normal. Mean pregnancy number is directly related with $\mathrm{BMI}(\mathrm{P}=0.07)$, showing that pregnancy is higher for obesity women than normal. Also, it is inversely related with INSU levels $(P=0.06)$, implying that pregnancy number rises as INSU levels decrease.

Also mean pregnancy number is inversely related with DPDF $(P=0.03)$, concluding that it rises as DPDF decreases. Note that the marginal effects GLUC, INSU levels, BMI, DW status, DPDF are all diabetic functions. The associations of the pregnancy numbers with these diabetic functions show that pregnancy is higher for diabetic women than normal. In addition, mean pregnancy number is inversely related with GLUC $\times$ Age $(P=0.02)$, or TSFT $\times \mathrm{BMI}(\mathrm{P}<0.01)$, implying that it rises as the joint effect GLUC $\times$ Age, or TSFT $\times$ Age decreases. Note that GLUC $\times$ Age and TSFT $\times$ BMI are joint diabetic interaction effects.

Similarly, from the dispersion model (Table 1), it is observed that variance of pregnancy number is directly related with the diabetic functions such as GLUC $(P=0.07)$, GLUC $\times$ TSFT $(P<0.01)$, DPDF $(P=0.01)$, INSU $\times B M I(P=0.15)$, while it is inversely related with the diabetic functions such as INSU $(P=0.07), G L U C \times D B P$ $(P=0.01)$, TSFT $\times$ DPDF $(P=0.03)$.

Further, variance of pregnancy number is directly related with the hypertension functions such as DBP $(P=0.04)$, DBP $\times$ TSFT $(P<0.01)$, while it is inversely related with the hypertension function GLUC $\times$ DBP $(P=0.01)$.

From Table 1, it is observed that there are some other nondiabetic and non-hypertension parameters such as Age, TSFT,
Age $\times$ TSFT are also related with the mean or dispersion of pregnancy's number. From the above, it is shown that pregnancy is associated with diabetic and hypertension functions. The present results can not be compared with the earlier findings as there is not any article which has focused on the pregnancy's associations with diabetic and hypertension parameters based on statistical modeling.

\section{Conclusions}

The current short article focuses on the relationships of pregnancy's number with some diabetic and hypertension functions based on joint log-normal models. The derived models are accepted using graphical diagnostic checking. It is derived herein that mean pregnancy's number is associated with the diabetic functions such as GLUC, INSU levels, diabetic history status, BMI, DPDF, while variance of pregnancy's number is associated with the many diabetic and hypertension functions. Pregnancy rate is higher for diabetic women than normal. The considered data set does not contain many other diabetic and hypertension functions such as $\mathrm{HbA1c}$, fasting glucose levels, random glucose levels, systolic blood pressure, basal blood pressure, maximum blood pressure etc. The current models are not compared with the joint gamma, or Weibull fitted models. In addition, the similar study is not done here for any other similar data set, as we have no similar data set in our hand. In our subsequent full research articles, we will examine the pregnancy's model based on joint gamma, log-normal, Weibull distributions. We propose to publish a full research article very soon by comparing both the log-normal and gamma models along with the detailed results in the near future.

\section{Conflict of interest:}

The author confirms that this article content has no conflict of interest.

\section{Acknowledgement:}

The authors are very much grateful for helping statistical analysis and interpretations to Dr. Jinseog Kim, Department of Applied Statistics, Dongguk University, Gyeongju, Republic of Korea.

\section{References}

1. Wei $Y, X u$ Q, Yang $H$, et al. (2019) Preconception diabetes mellitus and adverse pregnancy outcomes in over 6.4 million women: A population-based cohort study in China. PLoS Med 16(10): pp. e1002926.

2. Lee KW, Ching SM, Ramachandran V, et al. (2018) Prevalence and risk factors of gestational diabetes mellitus in Asia: a systematic review and meta-analysis. BMC Pregnancy and Childbirth 18(1): pp. 494.

3. Barker DJ (1995) Fetal origins of coronary heart disease. BMJ 311(6998): pp. 171-174.

4. Youngwanichsetha S (2020) Association of gestational diabetes and hypertensive disorders among pregnant women. Arch Clin Hypertens 6(1): pp. 13-14.

5. Carpenter MW (2007) Gestational diabetes, pregnancy hypertension, and late vascular disease. Diabetes Care 30(Supplement 2): pp. S246-S50.

6. Metzger BE, Coustan DR, Committee O (1998) Summary 
and recommendations of the fourth international workshopconference on gestational diabetes mellitus. Diabetes Care 21: pp. B161.

7. Wendland EM, Torloni MR, Falavigna M, et al. (2012) Gestational diabetes and pregnancy outcomes-a systematic review of the World Health Organization (WHO) and the International Association of Diabetes in Pregnancy study groups (IADPSG) diagnostic criteria. BMC Pregnancy Childbirth 12(1): pp. 23.

8. Group HSCR (2008) Hyperglycemia and adverse pregnancy outcomes. N Engl J Med 358(19): pp. 1991-2002.

9. Hillier TA, Pedula KL, Vesco KK, et al. (2008) Excess gestational weight gain: modifying fetal macrosomia risk associated with maternal glucose. Obstet Gynecol 112(5): pp. 1007-1014.

10. Hauth J, Clifton R, Roberts J, et al. (2012) Maternal insulin resistance and preeclampsia. Obstet Anesth Dig 32(1): pp. 4243.

11. Das M, Ray M, Chakraborty P, et al. (2021) Teenage Induced Abortions Trends for New Zealand between 2000-2019. J Obst
Gynecol Surg 2(1): 1-6.

12. Das M (2021) Induced Abortion Trends for Very Young Girls in New Zealand for the Period 2000-2019. J Med: Stud Res 4: pp. 19.

13. Das M (2021) Induced Abortion Trends for Senior Women ( $\geq$ 35 Years Old) in New Zealand Between 2000-2019. Acta Sci Clin Case Rep 2(9): pp. 24-30.

14. Lee, Y, Nelder JA, PawitanY (2017) Generalized Linear Models with Random Effects (Unified Analysis via H-likelihood) (second edition). Chapman and Hall, London.

15. Das RN, Lee Y (2009) Log-normal versus gamma models for analyzing data from quality-improvement experiments. Quality Engineering 21(1): pp. 79-87.

16. Das RN (2014) Robust response surfaces, regression and positive data analyses. Chapman and Hall, London.

17. Das M, Ray M, Sarkar AK, et al. (2021) History of new world silver production trends between 1521-1810. J Econometr Stat 1(1): 43-59. 